UDC 517.9

\title{
NEW SOLVABILITY CONDITIONS FOR A NONLOCAL BOUNDARY-VALUE PROBLEM FOR NONLINEAR FUNCTIONAL DIFFERENTIAL EQUATIONS*
}

\section{ЗНОВІ УМОВИ РОЗВ'ЯЗНОСТІ НЕЛОКАЛЬНИХ ГРАНИЧНИХ ЗАДАЧ} ДЛЯ НЕЛІНІЙНИХ ФУНКЦІНАЛЬНО-ДИФЕРЕНЦІАЛЬНИХ РІВНЯНЬ

\section{Z. Opluštil}

Inst. Math.

Technická 2, 61669 Brno, Czech Republic

e-mail: oplustil@fme.vutbr.cz

New efficient conditions are obtained sufficient for the solvability as well as unique solvability of a nonlocal boundary-value problem for nonlinear functional differential equations.

Отримано нові ефрективні умови розв'язності, а також єдиної розв'язності нелокальних граничних задач для нелінійних функціонально-диференціальних рівнянь.

1. Introduction and notation. On the interval $[a, b]$, we consider the functional differential equation

$$
u^{\prime}(t)=F(u)(t)
$$

where $F: C([a, b] ; \mathbb{R}) \rightarrow L([a, b] ; \mathbb{R})$ is a continuous (in general) nonlinear operator. As usual, by a solution of this equation we understand an absolutely continuous function $u:[a, b] \rightarrow \mathbb{R}$ satisfying the equality (1) almost everywhere on $[a, b]$. Along with the equation (1), we consider the nonlocal boundary condition

$$
h(u)=\varphi(u),
$$

where $h: C([a, b] ; \mathbb{R}) \rightarrow \mathbb{R}$ is a (non-zero) linear bounded functional and $\varphi: C([a, b] ; \mathbb{R}) \rightarrow \mathbb{R}$ is a continuous (in general) nonlinear functional.

The question on the solvability of various types of initial and boundary-value problems for functional differential equations and their systems is a classical topic in the theory of differential equations (see, e.g., [1-11] and references therein). There is a lot of interesting general results but only a few efficient conditions is known, namely, in the case where the boundary condition considered is nonlocal. In [12], we studied the question on the unique solvability of the problem $(1),(2)$ in the linear case, i.e., in the case where the operator $F$ is linear and $\varphi \equiv$ Const. We found out that it is very useful to consider the boundary condition (2) as a nonlocal perturbation of the two-point condition

$$
u(a)-\lambda u(b)=\varphi(u)
$$

${ }^{*}$ Published results were acquired using the subsidization of the Ministry of Education, Youth and Sports of the Czech Republic, research plan MSM 0021630518 "Simulation modelling of mechatronic systems".

(c) Z. Opluštil, 2008

ISSN 1562-3076. Нелінійні коливання, 2008, m. 11, №3 
with $\lambda \in \mathbb{R}$. In this paper, the results stated in [3] concerning the problem (1), (3) are generalized, and new efficient conditions are thus found sufficient for the solvability and unique solvability of the problem (1), (2).

The following notation is used in the sequel.

1. $\mathbb{R}$ is the set of all real numbers, $\mathbb{R}_{+}=[0,+\infty[$.

2. $C([a, b] ; \mathbb{R})$ is the Banach space of continuous functions $v:[a, b] \rightarrow \mathbb{R}$ with the norm $\|v\|_{C}=\max \{|v(t)|: t \in[a, b]\}$.

3. $C\left([a, b] ; \mathbb{R}_{+}\right)=\{u \in C([a, b] ; \mathbb{R}): u(t) \geq 0$ for $t \in[a, b]\}$.

4. $L([a, b] ; \mathbb{R})$ is the Banach space of Lebesgue integrable functions $p:[a, b] \rightarrow \mathbb{R}$ with the norm $\|p\|_{L}=\int_{a}^{b}|p(s)| d s$.

5. $L\left([a, b] ; \mathbb{R}_{+}\right)=\{p \in L([a, b] ; \mathbb{R}): p(t) \geq 0$ for almost all $t \in[a, b]\}$.

6. $\mathcal{L}_{a b}$ is the set of linear operators $\ell: C([a, b] ; \mathbb{R}) \rightarrow L([a, b] ; \mathbb{R})$ for which there is a function $\eta \in L\left([a, b] ; \mathbb{R}_{+}\right)$such that

$$
|\ell(v)(t)| \leq \eta(t)\|v\|_{C} \quad \text { for a.e. } \quad t \in[a, b] \quad \text { and all } \quad v \in C([a, b] ; \mathbb{R}) .
$$

7. $P_{a b}$ is the set of operators $\ell \in \mathcal{L}_{a b}$ transforming the set $C\left([a, b] ; \mathbb{R}_{+}\right)$into the set $L\left([a, b] ; \mathbb{R}_{+}\right)$.

8. $F_{a b}$ is the set of linear bounded functionals $h: C([a, b] ; \mathbb{R}) \rightarrow \mathbb{R}$.

9. $P F_{a b}$ is the set of functionals $h \in F_{a b}$ transforming the set $C\left([a, b] ; \mathbb{R}_{+}\right)$into the set $\mathbb{R}_{+}$.

10. $\mathcal{B}_{h c}^{i}=\left\{u \in C([a, b] ; \mathbb{R}):(-1)^{i+1} h(u) \operatorname{sgn}((2-i) u(a)+(i-1) u(b)) \leq c\right\}$, where $h \in F_{a b}, c \in \mathbb{R}, i=1,2$.

11. $K([a, b] \times A ; B)$, where $A, B \subseteq \mathbb{R}$, is the set of function $f:[a, b] \times A \rightarrow B$ satisfying the Carathéodory conditions, i.e., $f(\cdot, x):[a, b] \rightarrow B$ is a measurable function for all $x \in A, f(t, \cdot)$ : $A \rightarrow B$ is a continuous function for almost every $t \in[a, b]$, and for every $r>0$ there exists $q_{r} \in L\left([a, b] ; \mathbb{R}_{+}\right)$such that

$$
|f(t, x)| \leq q_{r}(t) \text { for a.e. } t \in[a, b] \text { and all } x \in A,|x| \leq r .
$$

2. Main results. As it was said above, the boundary condition (2) is considered as a non-local perturbation of the two-point condition (3). Therefore, we assume in the sequel that the linear functional $h$ appearing in (2) is defined by the formula

$$
h(v)=u(a)-\lambda v(b)-h_{0}(v)+h_{1}(v) \quad \text { for } \quad v \in C([a, b] ; \mathbb{R}),
$$

where $\lambda>0$ and $h_{0}, h_{1} \in P F_{a b}$. Moreover, the following assumptions are used:

$\left(H_{1}\right) F: C([a, b] ; \mathbb{R}) \rightarrow L([a, b] ; \mathbb{R})$ is a continuous operator such that the relation

$$
\sup \left\{|F(v)(\cdot)|: v \in C([a, b] ; \mathbb{R}),\|v\|_{C} \leq r\right\} \in L\left([a, b] ; \mathbb{R}_{+}\right)
$$

is satisfied for every $r>0$.

$\left(H_{2}\right) \varphi: C([a, b] ; \mathbb{R}) \rightarrow \mathbb{R}$ is a continuous functional such that the condition

$$
\sup \left\{|\varphi(v)|: v \in C([a, b] ; \mathbb{R}),\|v\|_{C} \leq r\right\}<+\infty
$$

holds for every $r>0$. 
Before formulation of the main results we introduce the following notation. Having $\lambda>0$ and $h \in F_{a b}$, we put

$$
\begin{aligned}
& \alpha(\lambda, h)=(1-h(1)) \min \left\{1, \frac{1}{\lambda}\right\}, \\
& \beta(\lambda, h)=(\lambda-h(1)) \min \left\{1, \frac{1}{\lambda}\right\} .
\end{aligned}
$$

Moreover, for any functional $h$ given by the formula (4), we define the function $\omega_{0}(\cdot ; h)$ by setting

$$
\omega_{0}(x ; h)=\left\{\begin{array}{l}
\frac{\left(x+\frac{1}{\lambda} h_{0}(1)\right)\left(1-h_{0}(1)\right)}{1-h_{0}(1)-x}-\left(\frac{1}{\lambda} h_{1}(1)+\frac{1-\lambda}{\lambda}\right) \\
\text { if } \quad \lambda \leq 1,\left(1-\lambda+h_{1}(1)\right) x<(1-h(1))\left(1-h_{0}(1)\right), \\
\frac{\left(x+h_{0}(1)\right)\left(1-h_{0}(1)\right)}{1-h_{0}(1)-x}-\left(h_{1}(1)+1-\lambda\right) \\
\text { if } \quad \lambda \leq 1,\left(1-\lambda+h_{1}(1)\right) x \geq(1-h(1))\left(1-h_{0}(1)\right), \\
\frac{\left(x+\lambda-1+h_{0}(1)\right)\left(1-h_{0}(1)\right)}{1-h_{0}(1)-\lambda x}-h_{1}(1) \\
\text { if } \quad \lambda>1, \lambda h_{1}(1) x<(1-h(1))\left(1-h_{0}(1)\right), \\
\frac{\left(x+\frac{\lambda-1}{\lambda}+\frac{1}{\lambda} h_{0}(1)\right)\left(1-h_{0}(1)\right)}{1-h_{0}(1)-\lambda x}-\frac{1}{\lambda} h_{1}(1) \\
\text { if } \quad \lambda>1, \lambda h_{1}(1) x \geq(1-h(1))\left(1-h_{0}(1)\right) .
\end{array}\right.
$$

In this section, we formulate all the results, the proofs are postponed till Section 5 below.

Theorem 1. Let $c \in \mathbb{R}_{+}$, the assumptions $\left(H_{1}\right)$ and $\left(H_{2}\right)$ be satisfied, and let the functional $h$ be defined by the formula (4), where $\lambda>0$ and $h_{0}, h_{1} \in P F_{a b}$ are such that

$$
\begin{gathered}
h(1) \geq 0, \\
h_{0}(1)<1, \quad h_{1}(1) \leq \lambda .
\end{gathered}
$$

Let, moreover, the condition

$$
\varphi(v) \operatorname{sgn} v(a) \leq c \quad \text { for } \quad v \in C([a, b] ; \mathbb{R})
$$

be fulfilled and there exist

$$
\ell_{0}, \ell_{1} \in P_{a b}
$$

such that, on the set $\mathcal{B}_{h c}^{1}([a, b] ; \mathbb{R})$, the inequality

$$
\left(F(v)(t)-\ell_{0}(v)(t)+\ell_{1}(v)(t)\right) \operatorname{sgn} v(t) \leq q\left(t,\|v\|_{C}\right) \quad \text { for a.e. } \quad t \in[a, b]
$$


holds, where the function $q \in K\left([a, b] \times \mathbb{R}_{+} ; \mathbb{R}_{+}\right)$satisfies

$$
\lim _{x \rightarrow+\infty} \frac{1}{x} \int_{a}^{b} q(s, x) d s=0 .
$$

If, in addition,

$$
\begin{gathered}
\left\|\ell_{0}(1)\right\|_{L}<\alpha\left(\lambda, h_{0}\right), \\
\omega_{0}\left(\left\|\ell_{0}(1)\right\|_{L} ; h\right)<\left\|\ell_{1}(1)\right\|_{L}<2 \sqrt{\alpha\left(\lambda, h_{0}\right)-\left\|\ell_{0}(1)\right\|_{L}}-h_{1}(1) \min \left\{1, \frac{1}{\lambda}\right\}
\end{gathered}
$$

then the problem (1), (2) has at least one solution.

Theorem 2. Let $c \in \mathbb{R}_{+}$, the assumptions $\left(H_{1}\right)$ and $\left(H_{2}\right)$ be satisfied, and let the functional $h$ be defined by the formula (4), where $\lambda>0$ and $h_{0}, h_{1} \in P F_{a b}$ satisfy the relations (7) and (8). Let moreover, the condition

$$
\varphi(v) \operatorname{sgn} v(b) \geq-c \quad \text { for } \quad v \in C([a, b] ; \mathbb{R})
$$

be fulfilled and there exist $\ell_{0}, \ell_{1} \in P_{a b}$ such that, on the set $\mathcal{B}_{h c}^{2}([a, b] ; \mathbb{R})$, the inequality

$$
\left(F(v)(t)-\ell_{0}(v)(t)+\ell_{1}(v)(t)\right) \operatorname{sgn} v(t) \geq-q\left(t,\|v\|_{C}\right) \quad \text { for a.e. } \quad t \in[a, b]
$$

holds, where the function $q \in K\left([a, b] \times \mathbb{R}_{+} ; \mathbb{R}_{+}\right)$satisfies (12). If, in addition,

$$
\left\|\ell_{1}(1)\right\|_{L}<\beta\left(\lambda, h_{1}\right)
$$

and

$$
\begin{aligned}
\frac{\alpha\left(\lambda, h_{0}\right)}{\beta\left(\lambda, h_{1}\right)-\left\|\ell_{1}(1)\right\|_{L}}-1 & <\left\|\ell_{0}(1)\right\|< \\
& <2 \sqrt{\beta\left(\lambda, h_{1}\right)-\left\|\ell_{0}(1)\right\|_{L}}-h_{0}(1) \min \left\{1, \frac{1}{\lambda}\right\}
\end{aligned}
$$

then the problem (1), (2) has at least one solution.

Now we establish theorems concerning the unique solvability of the problem (1), (2).

Theorem 3. Let the assumptions $\left(H_{1}\right)$ and $\left(H_{2}\right)$ be satisfied and a functional $h$ be defined by the formula (4), where $\lambda>0$ and $h_{0}, h_{1} \in P F_{a b}$ satisfy the relations (7) and (8). Let, moreover, the condition

$$
(\varphi(v)-\varphi(w)) \operatorname{sgn}(v(a)-w(a)) \leq 0 \quad \text { for } \quad v, w \in C([a, b] ; \mathbb{R})
$$


be fulfilled and there exist $\ell_{0}, \ell_{1} \in P_{a b}$ such that, on the set $\mathcal{B}_{h c}^{1}([a, b] ; \mathbb{R})$ with $c=|\varphi(0)|$, the inequality

$$
\left(F(v)(t)-F(w)(t)-\ell_{0}(v-w)(t)+\ell_{1}(v-w)(t)\right) \operatorname{sgn}(v(t)-w(t)) \leq 0 \quad \text { for a.e. } \quad t \in[a, b]
$$

holds. If, in addition, the conditions (13) and (14) are fulfilled then the problem (1), (2) is uniquely solvable.

Theorem 4. Let the assumptions $\left(H_{1}\right)$ and $\left(H_{2}\right)$ be satisfied and the functional $h$ be defined by the formula (4), where $\lambda>0$ and $h_{0}, h_{1} \in P F_{a b}$ satisfy the relations (7) and (8). Let, moreover, the condition

$$
(\varphi(v)-\varphi(w)) \operatorname{sgn}(v(b)-w(b)) \geq 0 \quad \text { for } \quad v, w \in C([a, b] ; \mathbb{R})
$$

be fulfilled and there exist $\ell_{0}, \ell_{1} \in P_{a b}$ such that, on the set $\mathcal{B}_{h c}^{2}([a, b] ; \mathbb{R})$ with $c=|\varphi(0)|$, the inequality

$$
\left(F(v)(t)-F(w)(t)-\ell_{0}(v-w)(t)+\ell_{1}(v-w)(t)\right) \operatorname{sgn}(v(t)-w(t)) \geq 0 \quad \text { for a.e. } \quad t \in[a, b]
$$

holds. If, in addition, the conditions (15) and (16) are fulfilled then the problem (1), (2) is uniquely solvable.

Remark 1. Let the functional $h$ be defined by the formula (4), where $\lambda>0$ and $h_{0}, h_{1} \in$ $\in P F_{a b}$. Define the operator $\psi: L([a, b] ; \mathbb{R}) \rightarrow L([a, b] ; \mathbb{R})$ by setting

$$
\psi(z)(t) \stackrel{\mathrm{df}}{=} z(a+b-t), \quad t \in[a, b],
$$

for an arbitrary $z \in L([a, b] ; \mathbb{R})$. Let $\omega$ be the restriction of $\psi$ to the space $C([a, b] ; \mathbb{R})$, and

$$
\begin{gathered}
\widehat{F}(z)(t) \stackrel{\text { df }}{=}-\psi(F(\omega(z)))(t) \quad \text { for a.e. } \quad t \in[a, b] \quad \text { and all } \quad z \in C([a, b] ; \mathbb{R}), \\
\widehat{h}(z) \stackrel{\text { df }}{=} z(a)-\frac{1}{\lambda} z(b)+\frac{1}{\lambda} h_{0}(\omega(z))-\frac{1}{\lambda} h_{1}(\omega(z)) \quad \text { for } \quad z \in C([a, b] ; \mathbb{R}), \\
\widehat{\varphi}(z) \stackrel{\text { df }}{=}-\frac{1}{\lambda} \varphi(\omega(z)) \text { for } z \in C([a, b] ; \mathbb{R}) .
\end{gathered}
$$

It is not difficult to verify that if $u$ is a solution to the problem (1), (2) then the function $v \stackrel{\mathrm{df}}{=} \omega(u)$ is a solution to the problem

$$
v^{\prime}(t)=\widehat{F}(v)(t), \quad \widehat{h}(v)=\widehat{\varphi}(v),
$$

and vice versa, if $v$ is a solution to the problem (17) then the function $u \stackrel{\mathrm{df}}{=} \omega(v)$ is a solution to the problem (1), (2).

Therefore, using the above transformation, we can immediately derive conditions for the solvability and unique solvability of the problem (1), (2) in the case where $h(1) \leq 0$ (we do not formulate them here in detail). 
3. An example. As an example, on the interval $[0,1]$, we consider the integro-differential equation

$$
u^{\prime}(t)=d \cos (2 \pi t) \int_{0}^{1} \frac{u(\tau(s))}{\sqrt{s}} d s-g_{1}(t) u(t) e^{u^{2}(\omega(t))}+g_{2}(t)|u(t)|^{\nu}
$$

subjected to the nonlocal boundary condition

$$
u(0)=\frac{1}{2} u(1)+k \int_{0}^{1} \sin (2 \pi s) u(s) d s-u(0) e^{u(1 / 4)}+\operatorname{arctg} u\left(\frac{1}{2}\right)
$$

where $d, k \in \mathbb{R}_{+}, \nu \in\left[0,1\left[, g_{1}, g_{2} \in L([0,1] ; \mathbb{R})\right.\right.$, and $\tau, \omega:[0,1] \rightarrow[0,1]$ are measurable functions.

Theorem 1 yields the following corollary.

Corollary 1. Let the function $g_{1}$ be nonnegative on $[0,1]$ and the numbers $d$ and $k$ satisfy

$$
k \leq \frac{\pi}{2}, \quad d<\frac{\pi-k}{2}
$$

and

$$
\frac{(d+k)(\pi-k)}{\pi-k-2 d}-\left(k+\frac{\pi}{2}\right)<d<2 \sqrt{\pi(\pi-k-2 d)}-\frac{k}{2} .
$$

Then the problem (18), (19) has at least one solution.

4. Auxiliary propositions. The main results are proved using a lemma on a priory estimate stated in [6] by Kiguradze and Pưža. This lemma can be formulated as follows.

Lemma 1 ([6], Corollary 2). Let there exist a positive number $\rho$ and an operator $\ell \in \mathcal{L}_{a b}$ such that homogeneous problem

$$
u^{\prime}(t)=\ell(u)(t), \quad h(u)=0
$$

has only the trivial solution, and, for every $\delta \in] 0,1[$, an arbitrary function $u \in \widetilde{C}([a, b] ; \mathbb{R})$ satisfying the relations

$$
u^{\prime}(t)=\ell(u)(t)+\delta[F(u)(t)-\ell(u)(t)] \quad \text { for a.e. } \quad t \in[a, b], \quad h(u)=\delta \varphi(u)
$$

admits the estimate

$$
\|u\|_{C} \leq \rho .
$$

Then the problem (1), (2) has at least one solution. 
Definition 1. Let $i \in\{1,2\}, h \in F_{a b}$. We say that an operator $\ell \in \mathcal{L}_{a b}$ belongs to the set $\mathcal{U}_{i}(h)$, if there exists $r>0$ such that, for arbitrary $q^{*} \in L\left([a, b] ; \mathbb{R}_{+}\right)$and $c \in \mathbb{R}_{+}$, every function $u \in \widetilde{C}([a, b] ; \mathbb{R})$ satisfying the inequalities

$$
\begin{gathered}
(-1)^{i+1} h(u) \operatorname{sgn}((2-i) u(a)+(i-1) u(b)) \leq c, \\
(-1)^{i+1}\left(u^{\prime}(t)-\ell(u)(t)\right) \operatorname{sgn} u(t) \leq q^{*}(t) \quad \text { for a.e. } \quad t \in[a, b]
\end{gathered}
$$

admits the estimate

$$
\|u\|_{C} \leq r\left(c+\left\|q^{*}\right\|_{L}\right)
$$

Lemma 2. Let $i \in\{1,2\}, c \in \mathbb{R}_{+}$, the assumptions $\left(H_{1}\right)$ and $\left(H_{2}\right)$ be satisfied, and

$$
(-1)^{i+1} \varphi(v) \operatorname{sgn}((2-i) v(a)+(i-1) v(b)) \leq c \quad \text { for } v \in C([a, b] ; \mathbb{R}) .
$$

Let, moreover, there exist $\ell \in \mathcal{U}_{i}(h)$ such that, on the set $\mathcal{B}_{h c}^{i}([a, b] ; \mathbb{R})$, the inequality

$$
(-1)^{i+1}(F(v)(t)-\ell(v)(t)) \operatorname{sgn} v(t) \leq q\left(t,\|v\|_{C}\right) \quad \text { for a.e. } \quad t \in[a, b]
$$

is fulfilled. Then the problem (1), (2) has at least one solution.

Proof. First note that, due to the condition $\ell \in \mathcal{U}_{i}(h)$, the homogeneous problem (22) has only the trivial solution.

Let $r$ be the number appearing in Definition 1. According to (12), there exists $\rho>2 r c$ such that

$$
\frac{1}{x} \int_{a}^{b} q(s, x)<\frac{1}{2 r} \text { for } x>\rho .
$$

Now assume that a function $u \in \widetilde{C}([a, b] ; \mathbb{R})$ satisfies (23) for some $\delta \in] 0,1[$. Then, according to (28), $u$ satisfies inequality (25), i.e., $u \in \mathcal{B}_{h c}^{i}([a, b] ; \mathbb{R})$. By (29), we obtain that inequality (26) is fulfilled with $q^{*} \equiv q\left(\cdot,\|u\|_{C}\right)$. Hence, by virtue of the condition $\ell \in \mathcal{U}_{i}(\lambda)$ and the definition of the number $\rho$, we get the estimate (24).

Since $\rho$ depends neither on $u$ nor on $\delta$, it follows from Lemma 1 that the problem (1), (2) has at least one solution.

Lemma 3. Let $i \in\{1,2\}$, the assumptions $\left(H_{1}\right)$ and $\left(H_{2}\right)$ be satisfied, and let the relation

$$
(-1)^{i+1}\left(\varphi\left(u_{1}\right)-\varphi\left(u_{2}\right)\right) \operatorname{sgn}\left((2-i)\left(u_{1}(a)-u_{2}(a)\right)+(i-1)\left(u_{1}(b)-u_{2}(b)\right)\right) \leq 0
$$

hold for every $u_{1}, u_{2} \in C([a, b] ; \mathbb{R})$. Let, moreover, there exist $\ell \in U_{i}(\lambda)$ such that, on the set $\mathcal{B}_{h c}^{i}([a, b] ; \mathbb{R})$ with $c=|\varphi(0)|$, the inequality

$$
(-1)^{i+1}\left(F\left(u_{1}\right)(t)-F\left(u_{2}\right)(t)-\ell\left(u_{1}-u_{2}\right)(t)\right) \operatorname{sgn}\left(u_{1}(t)-u_{2}(t)\right) \leq 0
$$

is fulfilled for a.e. $t \in[a, b]$. Then the problem (1), (2) is uniquely solvable. 
Proof. It follows from the condition (30) that the inequality (28) is fulfilled, where $c=$ $=|\varphi(0)|$. Using (31), we get that, on the set $\mathcal{B}_{h c}^{i}([a, b] ; \mathbb{R})$, the inequality (29) holds, where $q \equiv$ $\equiv|F(0)|$. Consequently, all the assumptions of Lemma 2 are fulfilled, and thus the problem (1), (2) has at least one solution. It remains to show that problem (1), (2) has at most one solution.

Let $u_{1}, u_{2}$ be arbitrary solutions of the problem (1), (2). Put $u(t)=u_{1}(t)-u_{2}(t)$ for $t \in$ $\in[a, b]$. Then, by virtue of (30) and (31), we get $u_{1}, u_{2} \in \mathcal{B}_{h c}^{i}([a, b] ; \mathbb{R})$ and

$$
\begin{gathered}
(-1)^{i+1} h(u) \operatorname{sgn}((2-i) u(a)+(i-1) u(b)) \leq 0, \\
(-1)^{i+1}\left(u^{\prime}(t)-\ell(u)(t)\right) \operatorname{sgn} u(t) \leq 0 \text { for a.e. } t \in[a, b] .
\end{gathered}
$$

The last relations, together with the assumption $\ell \in U_{i}(\lambda)$, result in $u \equiv 0$. Consequently, $u_{1} \equiv u_{2}$.

Lemma 4. Let the functional $h$ be defined by the formula (4), where $\lambda>0$ and $h_{0}, h_{1} \in$ $\in P F_{a b}$ satisfy the conditions (7) and (8). Let, moreover, the operator $\ell$ admit the representation $\ell=\ell_{0}-\ell_{1}$, where $\ell_{0}$ and $\ell_{1}$ are such that the conditions (10), (13), and (14) hold. Then $\ell$ belongs to the $\operatorname{set}_{1}(h)$.

Proof. Let $c \in \mathbb{R}_{+}, q^{*} \in L\left([a, b] ; \mathbb{R}_{+}\right)$, and $u \in \widetilde{C}([a, b] ; \mathbb{R})$ satisfy (25) and (26) with $i=1$. We shall show that (27) holds, where $r$ depends only on $\left\|\ell_{0}(1)\right\|_{L},\left\|\ell_{1}(1)\right\|_{L}, \lambda, h_{0}(1)$, and $h_{1}(1)$. It is clear that

$$
u^{\prime}(t)=\ell_{0}(u)(t)-\ell_{1}(u)(t)+\widetilde{q}(t) \quad \text { for a.e. } t \in[a, b],
$$

where

$$
\widetilde{q}(t)=u^{\prime}(t)-\ell(u)(t) \text { for a.e. } t \in[a, b] .
$$

From (25) and (26) we get

$$
\left(u(a)-\lambda u(b)-h_{0}(u)+h_{1}(u)\right) \operatorname{sgn} u(a) \leq c
$$

and

$$
\widetilde{q}(t) \operatorname{sgn} u(t) \leq q^{*}(t) \text { for a.e. } t \in[a, b] .
$$

First suppose that $u$ does not change its sign. Put

$$
\bar{M}=\max \{|u(t)|: t \in[a, b]\}, \quad \bar{m}=\min \{|u(t)|: t \in[a, b]\}
$$

and choose $t_{1}, t_{2} \in[a, b]$ such that $t_{1} \neq t_{2}$ and

$$
\left|u\left(t_{1}\right)\right|=\bar{M}, \quad\left|u\left(t_{2}\right)\right|=\bar{m} .
$$

It is clear that $\bar{M} \geq 0, \bar{m} \geq 0$, and either

$$
t_{1}<t_{2}
$$


or

$$
t_{1}>t_{2}
$$

Moreover, according to (10), (35), and (36), from (32) we obtain

$$
|u(t)|^{\prime} \leq \bar{M} \ell_{0}(1)(t)-\bar{m} \ell_{1}(1)(t)+q^{*}(t) \quad \text { for a.e. } t \in[a, b] .
$$

If $u(a)=0$ then $\bar{m}=0$ and the integration of (40) from $a$ to $t_{1}$, on account of (37), yields

$$
\bar{M} \leq \bar{M} \int_{a}^{t_{1}} \ell_{0}(1)(s) d s+\int_{a}^{t_{1}} q^{*}(s) d s .
$$

By virtue of (13), it follows from the last inequality that

$$
\|u\|_{C}=\bar{M} \leq r_{0}\left(\left\|q^{*}\right\|+c\right)
$$

where

$$
r_{0}=\left[1-\left\|\ell_{0}(1)\right\|_{L}\right]^{-1} .
$$

Consequently, the estimate (27) holds with $r=r_{0}$.

If $u(a) \neq 0$ then, according to (34), we obtain

$$
|u(a)| \leq \lambda|u(b)|+h_{0}(|u|)-h_{1}(|u|)+c,
$$

and thus

$$
|u(a)|-|u(b)| \leq h_{0}(|u|)-h_{1}(|u|)+|u(b)|(\lambda-1)+c
$$

and

$$
|u(a)|-|u(b)| \leq \frac{1}{\lambda} h_{0}(|u|)-\frac{1}{\lambda} h_{1}(|u|)+|u(a)| \frac{\lambda-1}{\lambda}+\frac{c}{\lambda} .
$$

Let first (38) hold. Then the integration of (40) from $a$ to $t_{1}$ and from $t_{2}$ to $b$ results in

$$
\begin{aligned}
& \bar{M}-|u(a)| \leq \bar{M} \int_{a}^{t_{1}} \ell_{0}(1)(s) d s+\int_{a}^{t_{1}} q^{*}(s) d s, \\
& |u(b)|-\bar{m} \leq \bar{M} \int_{t_{2}}^{b} \ell_{0}(1)(s) d s+\int_{t_{2}}^{b} q^{*}(s) d s .
\end{aligned}
$$

Summing two last inequalities, we get

$$
\bar{M}-\bar{m}+|u(b)|-|u(a)| \leq \bar{M} \int_{J} \ell_{0}(1)(s) d s+\int_{J} q^{*}(s) d s,
$$


where $J=\left[a, t_{1}\right] \cup\left[t_{2}, b\right]$.

Let $\lambda \leq 1$. Taking into account (10) and (41), we obtain from (43) that

$$
\bar{M}-\bar{m}-h_{0}(|u|)+h_{1}(|u|)-|u(b)|(\lambda-1)-c \leq \bar{M} \int_{a}^{b} \ell_{0}(1)(s) d s+\int_{a}^{b} q^{*}(s) d s .
$$

From the last inequality, according to (36), we get

$$
\bar{M}\left(1-h_{0}(1)\right)-\bar{m}\left(\lambda-h_{1}(1)\right)-c \leq \bar{M}\left\|\ell_{0}(1)\right\|_{L}+\left\|q^{*}\right\|_{L} .
$$

Due to (7), the last inequality implies

$$
(\bar{M}-\bar{m})\left(1-h_{0}(1)\right) \leq \bar{M}\left\|\ell_{0}(1)\right\|_{L}+\left\|q^{*}\right\|_{L}+c .
$$

Let $\lambda>1$. From (43), in view of (7), (42), and (36), it follows that

$$
\frac{1}{\lambda}(\bar{M}-\bar{m})\left(1-h_{0}(1)\right)-c \leq \bar{M}\left\|\ell_{0}(1)\right\|_{L}+\left\|q^{*}\right\|_{L}
$$

which, together with (44), yields

$$
\min \left\{1, \frac{1}{\lambda}\right\}(\bar{M}-\bar{m})\left(1-h_{0}(1)\right) \leq \bar{M}\left\|\ell_{0}(1)\right\|_{L}+\left\|q^{*}\right\|_{L}+c .
$$

Now suppose that (39) is fulfilled. Then the integration of (40) from $t_{2}$ to $t_{1}$, on account of (10) and (36), yields

$$
\min \left\{1, \frac{1}{\lambda}\right\}(\bar{M}-\bar{m})\left(1-h_{0}(1)\right)-c \leq \bar{M}-\bar{m} \leq \bar{M}\left\|\ell_{0}(1)\right\|_{L}+\left\|q^{*}\right\|_{L}
$$

Therefore, in both cases (38) and (39), the inequality

$$
\min \left\{1, \frac{1}{\lambda}\right\}(\bar{M}-\bar{m})\left(1-h_{0}(1)\right) \leq \bar{M}\left\|\ell_{0}(1)\right\|_{L}+\left\|q^{*}\right\|_{L}+c
$$

holds.

On the other hand, the integration of (40) from $a$ to $b$, yields

$$
|u(b)|-|u(a)| \leq \bar{M}\left\|\ell_{0}(1)\right\|_{L}-\bar{m}\left\|\ell_{1}(1)\right\|_{L}+\left\|q^{*}\right\|_{L} .
$$

Now we shall divide the discussion into the following four cases:

Case $(a): \lambda \leq 1$ and $\left(1-\lambda+h_{1}(1)\right)\left\|\ell_{0}(1)\right\|_{L}<(1-h(1))\left(1-h_{0}(1)\right)$. Using (42) and (36) in (46), we get

$$
\bar{m} \leq \frac{\bar{M}\left(h_{0}(1)+\lambda\left\|\ell_{0}(1)\right\|_{L}\right)+c+\lambda\left\|q^{*}\right\|_{L}}{\lambda\left\|\ell_{1}(1)\right\|_{L}+h_{1}(1)-\lambda+1} .
$$


On the other hand, (45) implies

$$
\bar{M}\left(1-h_{0}(1)-\left\|\ell_{0}(1)\right\|_{L}\right) \leq \bar{m}\left(1-h_{0}(1)\right)+c+\left\|q^{*}\right\|_{L} .
$$

Hence, on account of the first inequality in (14), we have

$$
\|u\|_{C}=\bar{M} \leq r_{1}\left(c+\left\|q^{*}\right\|_{L}\right)\left(\lambda\left\|\ell_{1}(1)\right\|_{L}+h_{1}(1)-h_{0}(1)-\lambda+2\right),
$$

where

$$
\begin{aligned}
r_{1}= & {\left[\left(1-h_{0}(1)-\left\|\ell_{0}(1)\right\|_{L}\right)\left(\lambda\left\|\ell_{1}(1)\right\|_{L}+h_{1}(1)-\lambda+1\right) \times\right.} \\
& \left.\times\left(-\left(1-h_{0}(1)\right)\left(h_{0}(1)+\lambda\left\|\ell_{0}(1)\right\|_{L}\right)\right)\right]^{-1}>0,
\end{aligned}
$$

and thus the estimate (27) holds, where $r$ is defined by

$$
r=r_{1}\left(\lambda\left\|\ell_{1}(1)\right\|_{L}+h_{1}(1)-h_{0}(1)-\lambda+2\right) .
$$

Case (b): $\lambda \leq 1$ and $\left(1-\lambda+h_{1}(1)\right)\left\|\ell_{0}(1)\right\|_{L} \geq(1-h(1))\left(1-h_{0}(1)\right)$. As above, (45) implies (47). If we use the estimate (41) in (46), according to (36), we obtain

$$
\bar{m} \leq \frac{\bar{M}\left(h_{0}(1)+\left\|\ell_{0}(1)\right\|_{L}\right)+c+\left\|q^{*}\right\|_{L}}{\left\|\ell_{1}(1)\right\|_{L}+h_{1}(1)-\lambda+1} .
$$

From the last inequality, the first inequality in (14), and (47) we get

$$
\|u\|_{C}=\bar{M} \leq r_{2}\left(c+\left\|q^{*}\right\|_{L}\right)\left(\left\|\ell_{1}(1)\right\|_{L}+h_{1}(1)-h_{0}(1)-\lambda+2\right),
$$

where

$$
\begin{aligned}
r_{2}= & {\left[\left(1-h_{0}(1)-\left\|\ell_{0}(1)\right\|_{L}\right)\left(\left\|\ell_{1}(1)\right\|_{L}+h_{1}(1)-\lambda+1\right) \times\right.} \\
& \left.\times\left(-\left(1-h_{0}(1)\right)\left(h_{0}(1)+\left\|\ell_{0}(1)\right\|_{L}\right)\right)\right]^{-1}>0,
\end{aligned}
$$

and thus the estimate (27) holds, where $r$ is defined by

$$
r=r_{2}\left(\lambda\left\|\ell_{0}(1)\right\|_{L}+h_{1}(1)-h_{0}(1)-\lambda+2\right) .
$$

Case (c): $\lambda>1$ and $\lambda h_{1}(1)\left\|\ell_{0}(1)\right\|_{L}<(1-h(1))\left(1-h_{0}(1)\right)$. From (46), in view of (41), and (36), it follows that

$$
\bar{m} \leq \frac{\bar{M}\left(h_{0}(1)+\left\|\ell_{0}(1)\right\|_{L}+\lambda-1\right)+c+\left\|q^{*}\right\|_{L}}{\left\|\ell_{1}(1)\right\|_{L}+h_{1}(1)} .
$$

On the other hand from the (45) we have

$$
\bar{M}\left(1-h_{0}(1)-\lambda\left\|\ell_{0}(1)\right\|_{L}\right) \leq \bar{m}\left(1-h_{0}(1)\right)+\lambda\left(c+\left\|q^{*}\right\|_{L}\right) .
$$


Hence, on account of (14), the relation

$$
\|u\|_{C}=\bar{M} \leq r_{3}\left(c+\left\|q^{*}\right\|_{L}\right)\left(\lambda\left\|\ell_{1}(1)\right\|_{L}+\lambda h_{1}(1)+1-h_{0}(1)\right),
$$

is satisfied, where

$$
\begin{aligned}
r_{3}= & {\left[\left(1-h_{0}(1)\right)\left(\left\|\ell_{1}(1)\right\|_{L}+h_{1}(1)-h_{0}(1)-\lambda+1-\left\|\ell_{0}(1)\right\|_{L}\right) \times\right.} \\
& \left.\times\left(-\lambda\left\|\ell_{0}(1)\right\|_{L}\left(h_{1}(1)+\left\|\ell_{1}(1)\right\|_{L}\right)\right)\right]^{-1}>0,
\end{aligned}
$$

and thus the estimate (27) holds, where $r$ is defined by

$$
r=r_{3}\left(\lambda\left\|\ell_{1}(1)\right\|_{L}+\lambda h_{1}(1)+1-h_{0}(1)\right) .
$$

Case $(d): \lambda>1$ and $\lambda h_{1}(1)\left\|\ell_{0}(1)\right\|_{L} \geq(1-h(1))\left(1-h_{0}(1)\right)$. As above, (45) yields (48). If we use estimate (42) in (46), according to (36), we obtain

$$
\bar{m} \leq \frac{\bar{M}\left(h_{0}(1)+\lambda\left\|\ell_{1}(1)\right\|_{L}+\lambda-1\right)+c+\lambda\left\|q^{*}\right\|_{L}}{\lambda\left\|\ell_{1}(1)\right\|_{L}+h_{1}(1)} .
$$

From the last inequality and (48), in view of (14), it follows that

$$
\|u\|_{C}=\bar{M} \leq r_{4} \lambda\left(c+\left\|q^{*}\right\|_{L}\right)\left(\lambda\left\|\ell_{1}(1)\right\|_{L}+h_{1}(1)+1-h_{0}(1)\right),
$$

where

$$
\begin{aligned}
r_{4}= & {\left[\left(1-h_{0}(1)-\lambda\left\|\ell_{0}(1)\right\|_{L}\right)\left(\lambda\left\|\ell_{1}(1)\right\|_{L}+h_{1}(1)\right) \times\right.} \\
& \left.\times\left(-\left(\lambda\left\|\ell_{0}(1)\right\|_{L}+\lambda-1+h_{0}(1)\right)\left(1-h_{0}(1)\right)\right)\right]^{-1}>0,
\end{aligned}
$$

and thus the estimate (27) holds, where $r$ is defined by

$$
r=r_{4} \lambda\left(\lambda\left\|\ell_{1}(1)\right\|_{L}+h_{1}(1)+1-h_{0}(1)\right) .
$$

Therefore, in all cases (a) - (d), the estimate (27) holds.

Now suppose that $u$ changes its sign. Put

$$
M=\max \{u(t): t \in[a, b]\}, \quad m=-\min \{u(t): t \in[a, b]\}
$$

and choose $t_{M}, t_{m} \in[a, b]$ such that

$$
u\left(t_{M}\right)=M, \quad u\left(t_{m}\right)=-m .
$$

Obviously, $M>0, m>0$, and either

$$
t_{m}<t_{M}
$$


or

$$
t_{m}>t_{M}
$$

First suppose that (51) holds. It is clear that there exists $\left.\alpha_{2} \in\right] t_{m}, t_{M}[$ such that

$$
u(t)>0 \quad \text { for } \quad \alpha_{2}<t \leq t_{M}, \quad u\left(\alpha_{2}\right)=0 .
$$

Let

$$
\alpha_{1}=\inf \left\{t \in\left[a, t_{m}\right]: u(s)<0 \text { for } t \leq s \leq t_{m}\right\}
$$

Obviously,

$$
u(t)<0 \quad \text { for } \quad \alpha_{1}<t \leq t_{m} \text { and } u\left(\alpha_{1}\right)=0 \text { if } \quad \alpha_{1}>a .
$$

Put

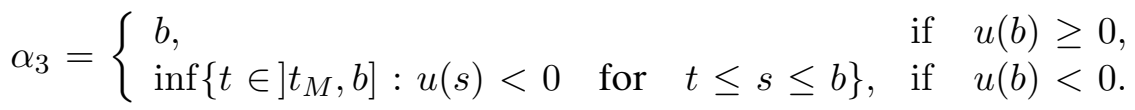

It is clear that if $\alpha_{3}<b$ then

$$
u(t)<0 \quad \text { for } \quad \alpha_{3}<t \leq b, \quad u\left(\alpha_{3}\right)=0 .
$$

The integration of (32) from $\alpha_{1}$ to $t_{m}$, from $\alpha_{2}$ to $t_{M}$, and from $\alpha_{3}$ to $b$, in view of (10), (35), (49), (50), and (53) - (55), yields

$$
\begin{gathered}
u\left(\alpha_{1}\right)+m \leq m \int_{\alpha_{1}}^{t_{m}} \ell_{0}(1)(s) d s+M \int_{\alpha_{1}}^{t_{m}} \ell_{1}(1)(s) d s+\int_{\alpha_{1}}^{t_{m}} q^{*}(s) d s, \\
M \leq M \int_{\alpha_{2}}^{t_{M}} \ell_{0}(1)(s) d s+m \int_{\alpha_{2}}^{t_{M}} \ell_{0}(1)(s) d s+\int_{\alpha_{2}}^{t_{M}} q^{*}(s) d s, \\
u\left(\alpha_{3}\right)-u(b) \leq m \int_{\alpha_{3}}^{b} \ell_{0}(1)(s) d s+M \int_{\alpha_{3}}^{b} \ell_{1}(1)(s) d s+\int_{\alpha_{3}}^{b} q^{*}(s) d s .
\end{gathered}
$$

If $u(b) \geq 0$ or $u(a) \geq 0$ then, according to (34), (49), (54), and the assumption $\lambda>0$, we obtain

$$
u\left(\alpha_{1}\right) \geq-c-m h_{0}(1)-M h_{1}(1)
$$

and thus from (56) we find

$-c-m h_{0}(1)-M h_{1}(1)+m \leq$

$$
\leq \max \{1, \lambda\}\left(m \int_{\alpha_{1}}^{t_{m}} \ell_{0}(1)(s) d s+M \int_{\alpha_{1}}^{t_{m}} \ell_{1}(1)(s) d s+\int_{\alpha_{1}}^{t_{m}} q^{*}(s) d s\right) .
$$


Hence,

$$
\begin{aligned}
\min \left\{1, \frac{1}{\lambda}\right\} & \left(-c-m h_{0}(1)-M h_{1}(1)+m\right) \leq \\
& \leq m \int_{J} \ell_{0}(1)(s) d s+M \int_{J} \ell_{1}(1)(s) d s+\int_{J} q^{*}(s) d s
\end{aligned}
$$

where $J=\left[\alpha_{1}, t_{m}\right] \cup\left[\alpha_{3}, b\right]$.

Let $u(b)<0$ and $u(a)<0$. Multiplying both sides of (58) by $\lambda$ and taking (55) into account, we get

$$
-\lambda u(b) \leq \lambda\left(m \int_{\alpha_{3}}^{b} \ell_{0}(1)(s) d s+M \int_{\alpha_{3}}^{b} \ell_{1}(1)(s) d s+\int_{\alpha_{3}}^{b} q^{*}(s) d s\right) .
$$

Summing the last inequality and (56), according to (34), (49), and (54), we can verify that the inequality (59) is fulfilled, where $J=\left[\alpha_{1}, t_{m}\right] \cup\left[\alpha_{3}, b\right]$.

From (57) and (59) we get

$$
\begin{gathered}
M\left(1-C_{1}\right) \leq m A_{1}+\left\|q^{*}\right\|_{L}+c \\
m\left(\alpha\left(\lambda, h_{0}\right)-D_{1}\right) \leq M\left(B_{1}+h_{1}(1) \min \left\{1, \frac{1}{\lambda}\right\}\right)+\left\|q^{*}\right\|_{L}+c
\end{gathered}
$$

where

$$
A_{1}=\int_{\alpha_{2}}^{t_{M}} \ell_{1}(1)(s) d s, \quad B_{1}=\int_{J} \ell_{1}(1)(s) d s
$$

and

$$
C_{1}=\int_{\alpha_{2}}^{t_{M}} \ell_{0}(1)(s) d s, \quad D_{1}=\int_{J} \ell_{0}(1)(s) d s .
$$


Due to (13), it is clear that $C_{1}<1$ and $D_{1}<\alpha\left(\lambda, h_{0}\right)$. Consequently, (60) and (61) imply

$$
\begin{aligned}
0 & <M\left(1-C_{1}\right)\left(\alpha\left(\lambda, h_{0}\right)-D_{1}\right) \leq \\
& \leq A_{1}\left[M\left(B_{1}+\min \left\{1, \frac{1}{\lambda}\right\} h_{1}(1)\right)+\left\|q^{*}\right\|_{L}+c\right]+\left(\left\|q^{*}\right\|_{L}+c\right)\left(\alpha\left(\lambda, h_{0}\right)-D_{1}\right) \leq \\
& \leq M A_{1}\left(B_{1}+\min \left\{1, \frac{1}{\lambda}\right\} h_{1}(1)\right)+\left(\left\|q^{*}\right\|_{L}+c\right)\left(1+\left\|\ell_{1}(1)\right\|_{L}\right), \\
0 & <m\left(\alpha\left(\lambda, h_{0}\right)-D_{1}\right)\left(1-C_{1}\right) \leq \\
& \leq\left(B_{1}+\min \left\{1, \frac{1}{\lambda}\right\} h_{1}(1)\right)\left(m A_{1}+\left\|q^{*}\right\|_{L}+c\right)+c+\left\|q^{*}\right\|_{L} \leq \\
& \leq m A_{1}\left(B_{1}+\min \left\{1, \frac{1}{\lambda}\right\} h_{1}(1)\right)+\left(\left\|q^{*}\right\|_{L}+c\right)\left(1+h_{1}(1)+\left\|\ell_{1}(1)\right\|_{L}\right) .
\end{aligned}
$$

Obviously,

$$
\left(1-C_{1}\right)\left(\alpha\left(\lambda, h_{0}\right)-D_{1}\right) \geq \alpha\left(\lambda, h_{0}\right)-\left(C_{1}+D_{1}\right) \geq \alpha\left(\lambda, h_{0}\right)-\left\|\ell_{0}(1)\right\|_{L}>0
$$

and

$$
\begin{aligned}
4 A_{1}\left(B_{1}+\min \left\{1, \frac{1}{\lambda}\right\} h_{1}(1)\right) & \leq\left(A_{1}+B_{1}+\min \left\{1, \frac{1}{\lambda}\right\} h_{1}(1)\right)^{2} \leq \\
& \leq\left(\left\|\ell_{1}(1)\right\|_{L}+\min \left\{1, \frac{1}{\lambda}\right\} h_{1}(1)\right)^{2} .
\end{aligned}
$$

By the last inequalities and the second inequality in (14), from (62) we get

$$
\begin{gathered}
M \leq r_{5}\left(\left\|\ell_{1}(1)\right\|_{L}+1\right)\left(\left\|q^{*}\right\|_{L}+c\right), \\
m \leq r_{5}\left(\left\|\ell_{1}(1)\right\|_{L}+1+h_{1}(1)\right)\left(\left\|q^{*}\right\|_{L}+c\right),
\end{gathered}
$$

where

$$
r_{5}=\left(\alpha\left(\lambda, h_{0}\right)-\left\|\ell_{0}(1)\right\|_{L}-\frac{\left(\left\|\ell_{1}(1)\right\|_{L}+\min \left\{1, \frac{1}{\lambda}\right\} h_{1}(1)\right)^{2}}{4}\right)^{-1} .
$$

Consequently, the estimate (27) holds, where $r$ is given by

$$
r=r_{5}\left(\left\|\ell_{1}(1)\right\|_{L}+1+h_{1}(1)\right) .
$$

If (52) holds, the validity of the estimate (27) can be proved analogously.

The lemma is proved.

Lemma 5. Let the functional $h$ be defined by the formula (4), where $\lambda>0$ and $h_{0}, h_{1} \in$ $\in P F_{a b}$ satisfy the conditions (7) and (8). Let, moreover, the operator $\ell$ admit the representation 
$\ell=\ell_{0}-\ell_{1}$, where $\ell_{0}$ and $\ell_{1}$ are such that the conditions (10), (15), and (16) hold. Then $\ell$ belongs to the $\operatorname{set}_{2}(h)$. i.e.,

Proof. Let $c \in \mathbb{R}_{+}, q^{*} \in L\left([a, b] ; \mathbb{R}_{+}\right)$, and $u \in \widetilde{C}([a, b] ; \mathbb{R})$ satisfy (25) and (26) with $i=2$,

$$
h(u) \operatorname{sgn} u(b) \geq-c
$$

and

$$
\left(u^{\prime}(t)-\ell(u)(t)\right) \operatorname{sgn} u(t) \geq-q^{*}(t) \text { for a.e. } t \in[a, b] .
$$

We shall show that (27) holds, where $r$ depends only on $\left\|\ell_{0}(1)\right\|_{L},\left\|\ell_{1}(1)\right\|_{L} \lambda, h_{0}(1)$, and $h_{1}(1)$. Obviously, $u$ satisfies (32), where $\widetilde{q}$ is defined by (33). Clearly,

$$
-\widetilde{q}(t) \operatorname{sgn} u(t) \leq q^{*}(t) \quad \text { for a.e. } \quad t \in[a, b]
$$

and

$$
\left(-u(a)+\lambda u(b)+h_{0}(u)-h_{1}(u)\right) \operatorname{sgn} u(b) \leq c .
$$

First suppose that $u$ does not change its sign. Define numbers $\bar{M}$ and $\bar{m}$ by (36) and choose $t_{1}, t_{2} \in[a, b]$ such that $t_{1} \neq t_{2}$ and (37) is fulfilled. Obviously, $\bar{M} \geq 0, \bar{m} \geq 0$, and either (38) or (39) holds. Moreover, according to (10), (36), and (63), from (32) we get

$$
-|u(t)|^{\prime} \leq \bar{M} \ell_{1}(1)(t)-\bar{m} \ell_{0}(1)(t)+q^{*}(t) \quad \text { for a.e. } t \in[a, b] .
$$

If $u(b)=0$ then $\bar{m}=0$ and the integration of (32) from $t_{1}$ to $b$, on account of (37), yields

$$
\bar{M} \leq \bar{M} \int_{t_{1}}^{b} \ell_{1}(1)(s) d s+\int_{t_{1}}^{b} q^{*}(s) d s .
$$

From the last inequality, in view of (15), it follows that

$$
\|u\|_{C}=\bar{M} \leq r_{0}\left(\left\|q^{*}\right\|+c\right)
$$

where

$$
r_{0}=\left[1-\left\|\ell_{1}(1)\right\|_{L}\right]^{-1} .
$$

Consequently, the estimate (27) holds with $r=r_{0}$.

If $u(b) \neq 0$ then, according to (64), we obtain

$$
\lambda|u(b)|-|u(a)| \leq h_{1}(|u|)-h_{0}(|u|)+c,
$$

and thus

$$
|u(b)|-|u(a)| \leq h_{1}(|u|)-h_{0}(|u|)+|u(b)|(1-\lambda)+c
$$


and

$$
\lambda(|u(b)|-|u(a)|) \leq h_{1}(|u|)-h_{0}(|u|)+|u(a)|(1-\lambda)+c .
$$

Let first (39) hold. The integration of (65) from $a$ to $t_{2}$ and from $t_{1}$ to $b$, in view of (10) and (37), results in

$$
|u(a)|-\bar{m} \leq \bar{M} \int_{a}^{t_{2}} \ell_{1}(1)(s) d s+\int_{a}^{t_{2}} q^{*}(s) d s
$$

and

$$
\bar{M}-|u(b)| \leq \bar{M} \int_{t_{1}}^{b} \ell_{1}(1)(s) d s+\int_{t_{1}}^{b} q^{*}(s) d s .
$$

Multiplying both sides of (69) by $\lambda$ and summing the last inequalities, in view of (10), (36), and (66), we get

$$
\bar{M}\left(\lambda-h_{1}(1)-\max \{1, \lambda\}\left\|\ell_{1}(1)\right\|_{L}\right) \leq \bar{m}\left(1-h_{0}(1)\right)+c+\max \{1, \lambda\}\left\|q^{*}\right\|_{L} .
$$

Hence, by virtue of (5) and (6), we obtain

$$
\bar{M}\left(\beta\left(\lambda, h_{1}\right)-\left\|\ell_{1}(1)\right\|_{L}\right) \leq \bar{m} \alpha\left(\lambda, h_{0}\right)+c+\left\|q^{*}\right\|_{L} .
$$

If (38) is fulfilled then the integration of (65) from $t_{1}$ to $t_{2}$, on account of (5), (6), and (7), yields

$$
\begin{aligned}
\bar{M} \beta\left(\lambda, h_{1}\right)-\bar{m} \alpha\left(\lambda, h_{0}\right) & \leq(\bar{M}-\bar{m})\left(\lambda-h_{1}(1)\right) \min \left\{1, \frac{1}{\lambda}\right\} \leq \\
& \leq \bar{M}-\bar{m} \leq \bar{M}\left\|\ell_{1}(1)\right\|_{L}+\left\|q^{*}\right\|_{L} .
\end{aligned}
$$

Therefore, in both cases (38) and (39), the inequality (70) holds.

On the other hand, the integration of (65) from $a$ to $b$ implies

$$
|u(a)|-|u(b)| \leq \bar{M}\left\|\ell_{1}(1)\right\|_{L}-\bar{m}\left\|\ell_{1}(1)\right\|_{L}+\left\|q^{*}\right\|_{L} .
$$

Hence, using (36), (67), and (68) in (71) we get

$$
\bar{m} \leq \frac{\bar{M}\left(1-\beta\left(\lambda, h_{1}\right)+\left\|\ell_{1}(1)\right\|_{L}\right)+\left\|q^{*}\right\|_{L}+c}{1+\left\|\ell_{0}(1)\right\|_{L}-\alpha\left(\lambda, h_{0}\right)} .
$$

From the last inequality and (70) we obtain

$$
\begin{aligned}
\bar{M}\left(\beta\left(\lambda, h_{1}\right)-\left\|\ell_{1}(1)\right\|_{L}\right) & \leq \\
& \leq \frac{\bar{M}\left(1-\beta\left(\lambda, h_{1}\right)+\left\|\ell_{1}(1)\right\|_{L}\right)+\left\|q^{*}\right\|_{L}+c}{1+\left\|\ell_{0}(1)\right\|_{L}-\alpha\left(\lambda, h_{0}\right)} \alpha\left(\lambda, h_{0}\right)+\left\|q^{*}\right\|_{L}+c .
\end{aligned}
$$


Thus, on account of (15) and the first inequality in (16), we have

$$
\|u\|_{C}=\bar{M} \leq r_{1}\left(c+\left\|q^{*}\right\|_{L}\right)\left(1+\left\|\ell_{0}(1)\right\|_{L}\right),
$$

where

$$
r_{1}=\left[\left(\beta\left(\lambda, h_{1}\right)-\left\|\ell_{1}(1)\right\|_{L}\right)\left(1+\left\|\ell_{0}(1)\right\|_{L}\right)-\alpha\left(\lambda, h_{0}\right)\right]^{-1}>0 .
$$

Therefore, the estimate (27) holds, where $r$ is defined by

$$
r=r_{1}\left(1+\left\|\ell_{0}(1)\right\|_{L}\right)
$$

Now suppose that $u$ changes its sign. Define numbers $M$ and $m$ by (49) and choose $t_{M}, t_{m} \in$ $\in[a, b]$ such that (50) is fulfilled. Obviously, $M>0, m>0$, and either (51) or (52) holds.

First suppose that (51) is fulfilled. It is clear that there exists $\left.\alpha_{1} \in\right] t_{m}, t_{M}[$ such that

$$
u(t)<0 \quad \text { for } t_{m} \leq t<\alpha_{1}, \quad u\left(\alpha_{1}\right)=0 .
$$

Let

$$
\alpha_{2}=\sup \left\{t \in[a, b]: u(s)>0 \text { for } t_{M} \leq s \leq t\right\}
$$

Obviously,

$$
u(t)>0 \quad \text { for } t_{M} \leq t<\alpha_{2} \quad \text { and } \quad u\left(\alpha_{2}\right)=0 \text { if } \quad \alpha_{2}<b
$$

Put

$$
\alpha_{3}= \begin{cases}a, & \text { if } u(a) \leq 0 \\ \sup \left\{t \in\left[a, t_{m}\right]: u(s)>0 \text { for } a \leq s \leq t\right\}, & \text { if } u(a)>0\end{cases}
$$

It is clear that if $\alpha_{3}>a$ then

$$
u(t)>0 \text { for } \quad a \leq t<\alpha_{3}, \quad u\left(\alpha_{3}\right)=0 .
$$

The integration of (32) from $t_{m}$ to $\alpha_{1}$, from $t_{M}$ to $\alpha_{2}$, and from $a$ to $\alpha_{3}$, in view of (10), (49), (63) and (72) - (74), yields

$$
\begin{gathered}
m \leq M \int_{t_{m}}^{\alpha_{1}} \ell_{0}(1)(s) d s+m \int_{t_{m}}^{\alpha_{1}} \ell_{1}(1)(s) d s+\int_{t_{m}}^{\alpha_{1}} q^{*}(s) d s, \\
M-u\left(\alpha_{2}\right) \leq M \int_{t_{M}}^{\alpha_{2}} \ell_{1}(1)(s) d s+m \int_{t_{M}}^{\alpha_{2}} \ell_{0}(1)(s) d s+\int_{t_{M}}^{\alpha_{2}} q^{*}(s) d s, \\
u(a)-u\left(\alpha_{3}\right) \leq M \int_{a}^{\alpha_{3}} \ell_{1}(1)(s) d s+m \int_{a}^{\alpha_{3}} \ell_{0}(1)(s) d s+\int_{a}^{\alpha_{3}} q^{*}(s) d s .
\end{gathered}
$$


If $u(b) \leq 0$ or $u(a) \leq 0$ then, according to (49), (64), and (73), we obtain

$$
\lambda u\left(\alpha_{2}\right) \leq m h_{0}(1)+M h_{1}(1)+c .
$$

Multiplying both sides of (76) by $\lambda$ and taking into account (10) and (78), we get

$$
\begin{aligned}
\lambda M-c-m h_{0}(1)-M h_{1}(1) & \leq \\
& \leq \lambda\left(M \int_{t_{M}}^{\alpha_{2}} \ell_{1}(1)(s) d s+m \int_{t_{M}}^{\alpha_{2}} \ell_{0}(1)(s) d s+\int_{t_{M}}^{\alpha_{2}} q^{*}(s) d s\right),
\end{aligned}
$$

and thus

$$
\begin{aligned}
& \min \left\{1, \frac{1}{\lambda}\right\}\left(\lambda M-c-m h_{0}(1)-M h_{1}(1)\right) \leq \\
& \leq M \int_{I} \ell_{1}(1)(s) d s+m \int_{I} \ell_{0}(1)(s) d s+\int_{I} q^{*}(s) d s,
\end{aligned}
$$

where $I=\left[a, \alpha_{3}\right] \cup\left[t_{M}, \alpha_{2}\right]$.

If $u(b)>0$ and $u(a)>0$ then multiplying both sides of (76) by $\lambda$ we get

$$
\lambda M-\lambda u\left(\alpha_{2}\right) \leq \lambda\left(M \int_{t_{M}}^{\alpha_{2}} \ell_{1}(1)(s) d s+m \int_{t_{M}}^{\alpha_{2}} \ell_{0}(1)(s) d s+\int_{t_{M}}^{\alpha_{2}} q^{*}(s) d s\right) .
$$

Summing the last inequality and (77), according to (49), (64), (73), and (74), we obtain that the inequality (80) with $I=\left[a, \alpha_{3}\right] \cup\left[t_{M}, \alpha_{2}\right]$ holds.

From (75) and (80) we get

$$
\begin{gathered}
m\left(1-A_{1}\right) \leq M C_{1}+\left\|q^{*}\right\|_{L}+c, \\
M\left(\beta\left(\lambda, h_{1}\right)-B_{1}\right) \leq m\left(\min \left\{1, \frac{1}{\lambda}\right\} h_{0}(1)+D_{1}\right)+\left\|q^{*}\right\|_{L}+c,
\end{gathered}
$$

where

$$
A_{1}=\int_{t_{m}}^{\alpha_{1}} \ell_{1}(1)(s) d s, \quad B_{1}=\int_{I} \ell_{1}(1)(s) d s
$$

and

$$
C_{1}=\int_{t_{m}}^{\alpha_{1}} \ell_{0}(1)(s) d s, \quad D_{1}=\int_{I} \ell_{0}(1)(s) d s .
$$

ISSN 1562-3076. Нелінійні коливання, 2008, m. 11, № 3 
Due to (15), it is clear that $A_{1}<1$ and $B_{1}<\beta\left(\lambda, h_{1}\right)$. Consequently, (81) and (82) imply

$$
\begin{aligned}
0 & <m\left(1-A_{1}\right)\left(\beta\left(\lambda, h_{1}\right)-B_{1}\right) \leq \\
& \leq m C_{1}\left(\min \left\{1, \frac{1}{\lambda}\right\} h_{0}(1)+D_{1}\right)+\left(\left\|q^{*}\right\|_{L}+c\right)\left(1+\left\|\ell_{0}(1)\right\|_{L}\right), \\
0 & <M\left(1-A_{1}\right)\left(\beta\left(\lambda, h_{1}\right)-B_{1}\right) \leq \\
& \leq M C_{1}\left(\min \left\{1, \frac{1}{\lambda}\right\} h_{0}(1)+D_{1}\right)+\left(\left\|q^{*}\right\|_{L}+c\right)\left(2+\left\|\ell_{0}(1)\right\|_{L}\right) .
\end{aligned}
$$

Obviously,

$$
\begin{aligned}
\left(1-A_{1}\right)\left(\beta\left(\lambda, h_{1}\right)-B_{1}\right) & \geq \beta\left(\lambda, h_{1}\right)-A_{1} \beta\left(\lambda, h_{1}\right)-B_{1} \geq \\
& \geq \beta\left(\lambda, h_{1}\right)-\left(A_{1}+B_{1}\right) \geq \beta\left(\lambda, h_{1}\right)-\left\|\ell_{1}(1)\right\|_{L}>0
\end{aligned}
$$

and

$$
\begin{aligned}
4 C_{1}\left(\min \left\{1, \frac{1}{\lambda}\right\} h_{0}(1)+D_{1}\right) & \leq\left(\min \left\{1, \frac{1}{\lambda}\right\} h_{0}(1)+C_{1}+D_{1}\right)^{2} \leq \\
& \leq\left(\min \left\{1, \frac{1}{\lambda}\right\} h_{0}(1)+\left\|\ell_{0}(1)\right\|_{L}\right)^{2}
\end{aligned}
$$

Hence, from the second inequality in (16) and (83) we obtain

$$
\begin{aligned}
& M \leq r_{2}\left(\left\|\ell_{0}(1)\right\|_{L}+2\right)\left(c+\left\|q^{*}\right\|_{L}\right), \\
& m \leq r_{2}\left(\left\|\ell_{0}(1)\right\|_{L}+1\right)\left(c+\left\|q^{*}\right\|_{L}\right),
\end{aligned}
$$

where

$$
r_{2}=\left[\beta\left(\lambda, h_{1}\right)-\left\|\ell_{1}(1)\right\|_{L}-\frac{\left(\left\|\ell_{0}(1)\right\|_{L}+\min \left\{1, \frac{1}{\lambda}\right\} h_{0}(1)\right)^{2}}{4}\right]^{-1} .
$$

Consequently, the estimate (27) holds, where $r$ is defined by

$$
r=r_{2}\left(\left\|\ell_{0}(1)\right\|_{L}+2\right)\left(c+\left\|q^{*}\right\|_{L}\right) .
$$

If (52) holds, the validity of the estimate (27) can be proved analogously.

The lemma is proved.

5. Proofs. Proof of Theorems 1 and 3. The validity of theorems follows from Lemmas 2, 3, and 4.

Proof of Theorem 2 and 4. The assertion of theorems can be derived from Lemmas 2, 3, and 5. 
Proof of Corollary 1. It is clear that (18), (19) is a particular case of (1), (2) in which $a=0$, $b=1$, and $F, h$, and $\varphi$ are defined by the formulae

$$
F(z)(t) \stackrel{\mathrm{df}}{=} d \cos (2 \pi t) \int_{0}^{1} \frac{z(\tau(s))}{\sqrt{s}} d s-g_{1}(t) z(t) e^{z^{2}(\omega(t))}+g_{2}(t)|z(t)|^{\nu}
$$

for a.e. $t \in[0,1]$ and all $z \in C([0,1] ; \mathbb{R})$ and

$$
h(z) \stackrel{\mathrm{df}}{=} z(0)-\frac{1}{2} z(1)-k \int_{0}^{1} \sin (2 \pi s) z(s) d s, \quad \varphi(z) \stackrel{\mathrm{df}}{=}-z(0) e^{z(1 / 4)}+\arctan z(1 / 2)
$$

for $z \in C([0,1] ; \mathbb{R})$, respectively. Moreover, the above-defined functional $h$ admits the representation (4), where $\lambda=1 / 2$ and

$$
h_{0}(z) \stackrel{\mathrm{df}}{=} k \int_{0}^{1} \max \{\sin (2 \pi s), 0\} z(s) d s, \quad h_{1}(z) \stackrel{\mathrm{df}}{=} k \int_{0}^{1} \max \{-\sin (2 \pi s), 0\} z(s) d s
$$

for $z \in C([0,1] ; \mathbb{R})$.

Now we put

$$
\begin{aligned}
& \ell_{0}(z)(t) \stackrel{\mathrm{df}}{=} d \max \{\cos (2 \pi t), 0\} \int_{0}^{1} \frac{z(\tau(s))}{\sqrt{s}} d s, \\
& \ell_{1}(z)(t) \stackrel{\mathrm{df}}{=} d \max \{-\cos (2 \pi t), 0\} \int_{0}^{1} \frac{z(\tau(s))}{\sqrt{s}} d s
\end{aligned}
$$

for a.e. $t \in[0,1]$ and all $z \in C([0,1] ; \mathbb{R})$.

It is easy to verify that $\ell_{0}, \ell_{1} \in P_{a b}, h_{0}, h_{1} \in P F_{a b}$, and

$$
\left\|\ell_{0}(1)\right\|_{L}=\left\|\ell_{1}(1)\right\|_{L}=\frac{2 d}{\pi}, \quad h_{0}(1)=h_{1}(1)=\frac{k}{\pi} .
$$

Therefore, in view of the assumptions (20) and (21), the conditions (7), (8), (13), and (14) of Theorem 1 are fulfilled. On the other hand, operators $F$ and $\varphi$ satisfy the assumptions $\left(H_{1}\right)$ and $\left(H_{2}\right)$, the relation (9) with $c=\pi / 2$ holds, and the inequality (11) is satisfied on the set $C([0,1] ; \mathbb{R})$, where $q(t, x)=\left|g_{2}(t)\right| x^{\nu}$ for a.e. $t \in[0,1]$ and all $x \in \mathbb{R}_{+}$.

Applying Theorem 1, we establish solvability of the problem (18), (19).

1. Azbelev N. V., Maksimov V. P., Rakhmatullina L. F. Introduction to the theory of functional differential equations (in Russian). - Moscow: Nauka, 1991.

2. Hakl R., Kiguradze I., Půža B. Upper and lower solutions of boundary-value problems for functional differential equations and theorems on functional differential inequalities // Georg. Math. -2000 . - 7, №. 3. P. $489-512$. 
3. Hakl R., Lomtatidze A., Šremr J. On a periodic type boundary-value problem for first order nonlinear functional differential equations // Nonlinear Anal. - 2002. - 51. - P. 425-447.

4. Hakl R., Lomtatidze A., Šremr J. Some boundary-value problems for first order scalar functional differential equations // Folia Fac. Sci. Natur. Univ. Masar. Brunensis, Brno. - 2002.

5. Hale J. Theory of functional differential equations. - New York etc.: Springer, 1977.

6. Kiguradze I., Půža B. On boundary-value problems for functional differential equations // Mem. Different. Equat. Math. Phys. - 1997. - 12. - P. 106-113.

7. Kiguradze I., Sokhadze Z. On the global solvability of the Cauchy problem for singular functional differential equations // Georg. Math. J. - 1997. - 4, № 4. - P. 355-373.

8. Kiguradze I., Sokhadze Z. On the uniqueness of a solution to the Cauchy problem for functional differential equations // Different. Equat. - 1995. - 31, № 12. - P. 1947-1958.

9. Kiguradze I. T., P ํ̆a B. Theorems of Conti-Opial type for nonlinear functional-differential equations // Ibid. - 1997. - 33, №. 2. - P. 184-193.

10. Kolmanovskii V., Myshkis A. Introduction to the theory and applications of functional differential equations. - Dordrecht etc.: Kluwer Acad. Publ., 1999.

11. Schwabik ̌́., Tvrdý M., Vejvoda O. Differential and integral equations: boundary-value problems and adjoints. - Praha: Academia, 1979.

12. Lomtatidze A., Opluštil Z., Šremr J. On a nonlocal boundary-value problem for first order linear functional differential equations // Mem. Different. Equat. Math. Phys. - 2007. - 41. - P. 69-85.

Received 19.11.07 